\title{
Self-related Process Residual Control Chart Based on Neural Network
}

\author{
Janli $\mathrm{Yu}^{1}$, Yang Han $^{2}$, Manxiang Miao ${ }^{1}$, Hongqi Huang ${ }^{1}$ \\ ${ }^{1}$.Zhengzhou Institute of Aeronautical Industry Management, Zhengzhou,450015,China \\ ${ }^{2}$ College of Management, National Taiwan University, Taibei, Taiwan
}

\begin{abstract}
The output data of the modern complex product manufacturing process shows the high correlation, leading to the output of the process deviates from the design target or increasing of false alarms when traditional Control Charts monitors the process. A kind of self-related process of residual control chart, which is based on the Neural network, is using Neural network to establish time series prediction model of the self-related process and apply this model to predict the output of the self-related process. Forming residual control chart with the output of the self-related process and the Neural network prediction residual to eliminate correlation of time series of the self-related process and implement statistical quality control of self-related process.
\end{abstract}

Keywords-neural network; self-related process; control chart.

\section{INTRODUCTION}

Modern industry frequently takecustomised production pattern of multispecies and small batch. That need to frequently replace raw material, adjust the setting monitor the process timely and automatic data collection, so the manufacturing process shows the high correlation. Structure of self-related process lead to the process output to deviates from the design target and the decrease of the quality of the products. At the same time, it also destroy the traditional quality control chart of independent assumption about process data and increase control chart of false alarm $^{[1]}$.Through forecasting process output, calculating process output and predicted residual error, and drawing the quality control of residual figure, it can achieve the goal of eliminating process autocorrelation ${ }^{[2]}$.

The key factors influencing the effect of residual control chart is the forecast accuracy of the self-related process. After making the time series undergo autocorrelation analysis, model identification and parameter estimation, the traditional Box- Jenkins time series prediction method, which is based on ARMA (p, q) model, can establish forecast model. BP (Back Propagation) Neural network has a fast speed of calculation and strong nonlinear function with approximation ability and generalization ability, so it is widely used in the time series prediction and the complex production process quality control ${ }^{[3-7]}$.

This paper use BP neural network to establish the time series prediction model of the self-related process, and apply this model to predict the output of the self-related process. Through drawing residual control chart of the self-related process, it can achieve quality control to the self-related process.

\section{THE TIME SERIES OF SELF-RELATED PROCESS}

About an anti-glare glass production, the time series of product percent of pass is labelled as $Z_{t}$ (as shown in figure 1).Using Minitab software to calculate and draw the autocorrelation function of time sequence (as shown in figure 2) can be seen that the process is an autocorrelation.

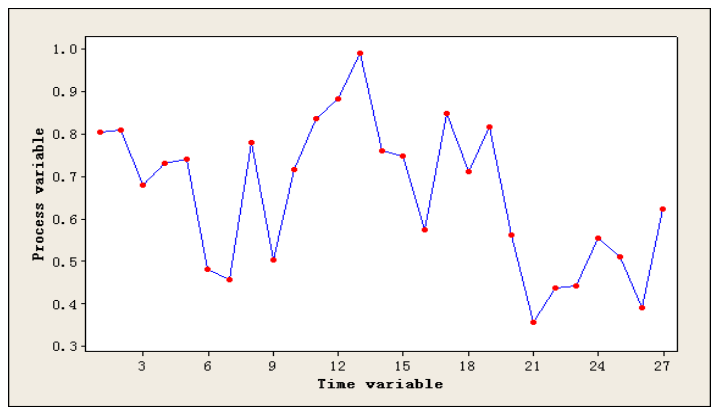

Figure 1.The time series of self-related process

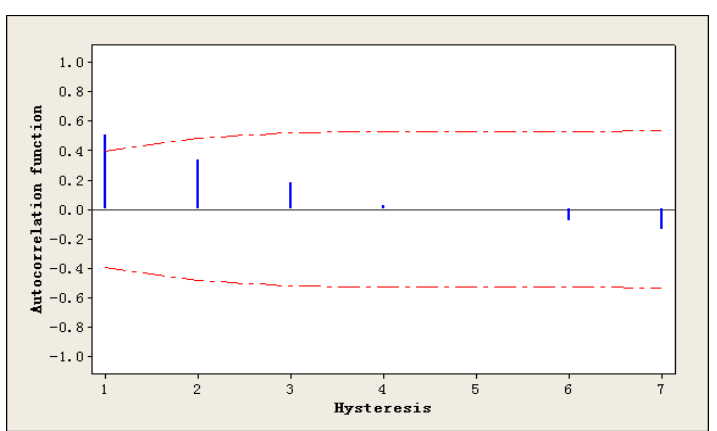

Figure 2. Figure of the autocorrelation function

III. PRINCIPLES OF BP (BACK PROPAGATION)NEURAL NETWORK

BP neural network is a multilayer feed forward neural networks trained by error back propagation algorithm. BP neural network can learn and store large amounts of input output mode mappings, however, mathematical equation of this mapping is not needed.

Learning method of BP neural network is the steepest descent method, which adjust weights and threshold values of neural network by back propagation, to minimizeerror 
sum squares of the BP neural network. Topology structure of BP neural network model consists of input layer, hidden layers and output layer (as shown in figure 3).

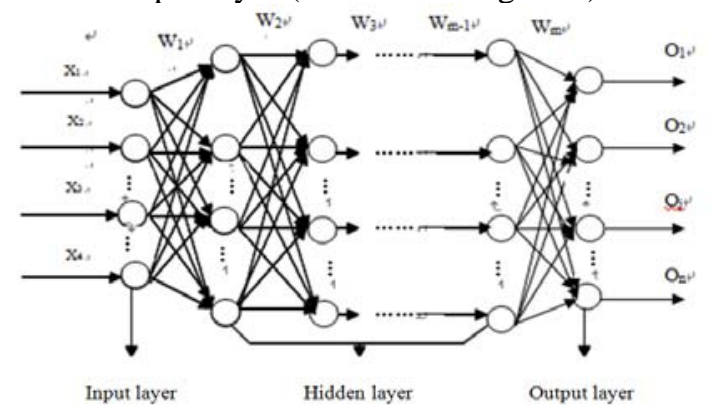

Figure 3. Structure chart

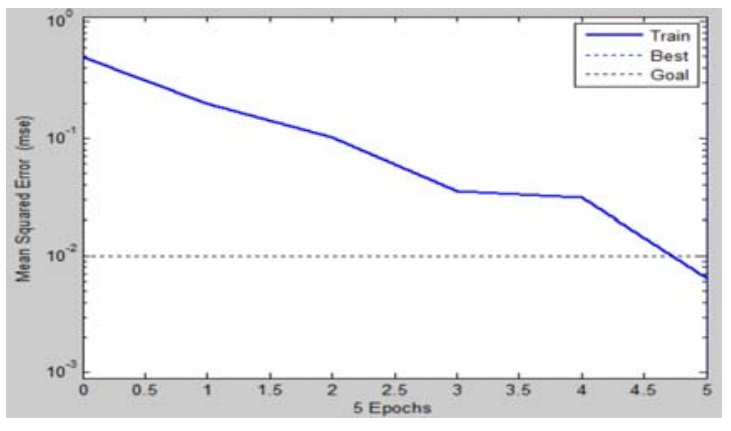

Figure 4. Training process

Forward-propagating and back propagation of BP neural network constitute its learning process. Forward-propagating means network computing, determine its output for given input; Back propagation is used for error propagation layer by layer, modify connection weight and threshold value. Algorithm step as below:

(1) Set variables and parameters of training network:

$X_{k}=\left[x_{k 1}, x_{k 2}, \ldots, x_{k P}\right],(k=1,2, \cdots, N)$ as input vector, i.e. training samples, total number of samples is $\mathrm{N} ; W_{P I}(n)=\left(w_{i j}\right)_{M \times I}$ as the nth iterative weights vector between the hidden layer and input layer $I$; $W_{P I}(n)=\left(w_{i j}\right)_{M \times I}$ as the nth iterative weights vector between the hidden layer $J$ and the hidden layer $I_{I}$; $W_{P I}(n)=\left(w_{i j}\right)_{M \times I}$ as the nth iterative weights vector between the hidden layer $J$ and output; $O_{k}(n)=\left[O_{k 1}(n), O_{k 2}(n), \cdots, O_{k N 3}(n)\right]$ as actual output of the nth iterative network; $d_{k}=\left[d_{k 1}, d_{k 2}, \cdots, d_{k N 3}\right],(k=1,2, \cdots, N)$ as expected output of trained network.

(2) Initialize the settings. Assigned to small random nonzero values for $W_{M I}(0), W_{I J}(0), W_{J N 3}(0)$.

(3) Afterwards, input samples $X_{k}, \quad n=0$.

(4) About input samples $X_{k}$, compute BP neural network input signal $u$ and output signal $v$ of every layer neuron in forward direction.

(5) Compute error $e$ according to actual output $O_{k}(n)$ and actual expected output $d_{k}$ by last step, judge whether meet the requirements, if meet the requirements, go to (8); If you don't meet ,go to (6).

(6) Judge if $n+1$ greater than the maximum iterative number. If greater than turn to(8), otherwise, compute local gradient $\delta$ of every layer for input sample $X_{k}$.

$$
\begin{aligned}
& \delta_{p}^{N 3}=O_{p}(n)\left(1-O_{p}(n)\right)\left(d_{p}(n)-O_{p}(n)\right), \\
& (p=1,2, \cdots, N 3) \\
& \delta_{j}^{J}=f^{\prime}\left(u_{j}^{J}(n)\right) \sum_{p=1}^{N 3} \delta_{p}^{N 3} w_{j p}(n), \\
& (j=1,2, \cdots, J) \\
& \delta_{i}^{I}=f^{\prime}\left(u_{i}^{I}(n)\right) \sum_{j=1}^{J} \delta_{j}^{J} w_{i j}(n),(i=1,2, \cdots, I)
\end{aligned}
$$

(7) Compute weight amendment $\Delta w$ according to formula below, and amend weight, $\eta$ is learning rate.

$$
\begin{aligned}
& \Delta w_{j p}(n)=\eta \delta_{p}^{N 3}(n) v_{j}^{J}(n), \\
& w_{j p}(n+1)=w_{j p}(n)+\Delta w_{j p}(n), \\
& (j=1,2, \cdots, J ; p=1,2, \cdots, P) ; \\
& \Delta w_{i j}(n)=\eta \delta_{j}^{J}(n) v_{i}^{I}(n), \\
& w_{i j}(n+1)=w_{i j}(n)+\Delta w_{i j}(n), \\
& (i=1,2, \cdots, I ; j=1,2, \cdots, J) ; \\
& \Delta w_{m i}(n)=\eta \delta_{i}^{I}(n) x_{k m}(n) \\
& w_{m i}(n+1)=w_{m i}(n)+\Delta w_{m i}(n) \\
& (m=1,2, \cdots, P ; i=1,2, \cdots, I)
\end{aligned}
$$

(8) Judge if whole samples be learned, if it is, turn to end, otherwise, go back (3).

\section{ESTABLISH THE BP NEURAL NETWORK PREDICTION MODEL}

Autocorrelation process of time series ${ }^{Z_{t}}$ for anti-glare glass, select $n=3$, and regard the former $n$ moment of process sequence value as a set of input and process sequence of values of the $n+1_{\text {moment as the output. BP }}$ neural network is the three-layer neural networks, included input layer, hidden layer and output layer .Besides, the input layer has 3 neurons, hidden layer has 10 neurons and output layer have 1 neurons. Choosing 0.001 as network training precision, using computer software of MATELA Bestablish and train the BP neural network prediction model. The neural network training process as shown in figure 4.

\section{V.USE NEURAL NETWORK TO PREDICT THE PROCESS OUTPUT}

Using BP neural network forecasting model forecast the process of autocorrelation sequence $Z_{t}$. The prediction result is shown in figure 5 .

In figure $5, \mathrm{z}$ is the originally actual process sequence value and the $\mathrm{z} 1$ is the predictive value of the BP neural network .From the experiment result shows in the graph, the BP neural network's predicted value about time series of the self-related process is well fitting with the actual values. 


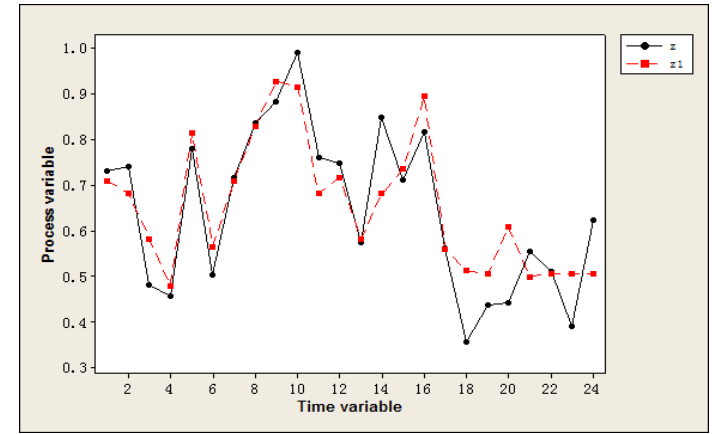

Figure 5. Prediction results

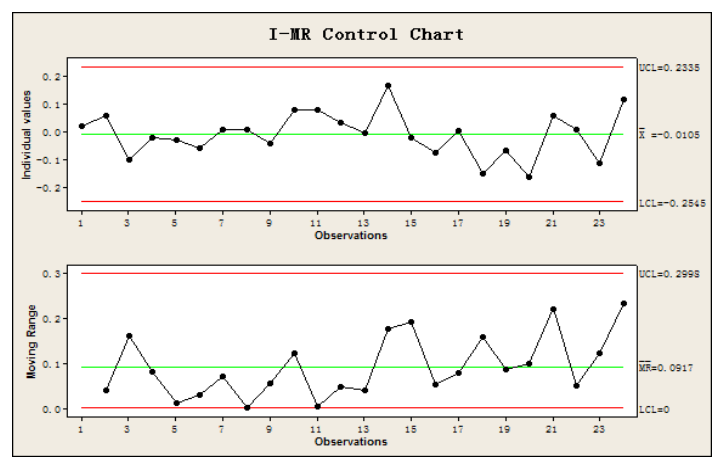

Figure 6. I-MR Control Chart

\section{RESIDUAL CONTROL CHART OF THE SELF-RELATED PROCESS}

Draw I-MR control chart of the self-related process residual by facilitating MINITAB computer software, as shown in figure 6.Follow the diagram, it can be seen that the anti-glare glass production process in a statistical stable state. In the process of production, therefore, it can apply section 4 of the BP neural network model established to forecast the process output, and then calculate residual value and I-MR between actual output values and predicted value of production process. Extending control line of figure 6 to make control moment of the control chart, and describing residual value and Moving Rang value on the control moment of the control chart, statistical quality control of the anti-glare glass's production process can be realized.

\section{CONCLUSIONS}

Autocorrelation process destroy traditional quality control chart of process data independence assumption and increase control chart of false alarm. BP Neural network has a strong approximation ability and generalization ability of nonlinear function. Using BP neural network to establish product percent of pass of time series forecasting model of anti-glare glass, it can accurately predict the actual value of the process. As a result, drawing the control chart about the actual value and predictive value of $\mathrm{x}-\mathrm{MR}$ of the residual, the results show that the Moving Rang value and the residual value are within the line of control chart, and anti-dazzle glass production process is in a statistically stable state. To extend the control line of control chart, the production process of control moment of the control chart is available.

\section{ACKNOWLEDGEMENTS}

This work is supported by the Natural Science Foundation of Henan Province under Grant No.142102210077, No.142102210105, and is supported by the Natural Science Foundation of Zhengzhou City under GrantNo.131PPTGG418-2

\section{REFERENCES}

1] Matos A. S, Requeijo J. G., Pereira Z. L. Integration of Engineering Process Control and Statistical Control in Pulp and Paper Industry [J]. COMPUTER AIDED CHEMICAL ENGINEERING, 2008, 25(3):399.

[2] CHU Wei, SUN Shudong, YU Xiaoyi. Study on the integration of SPC/EPC and its key technologyies [J]. Computer Applications,2007,27(1):28-30.

[3] Corzo G., Solomatine D. Baseflow separation techniques for modular artificial neural network modelling in flow forecasting/Techniques de separation de l'ecoulement de base pour la modelisationmodulaire par reseau de neuronesartificiels a vocation de prevision de debit[J]. Hydrological Sciences Journal/Journal des Sciences Hydrologiques, 2007, 52(3):491-507.

[4] Lu Y, Lang R. A Hybrid Prediction Method Combining RBF Neural Network and FAR Model [J]. LECTURE NOTES IN COMPUTER SCIENCE, 2007, 44(26):598.

[5] Jain A, Kumar A. M. Hybrid neural network models for hydrologic time series forecasting [J]. Applied Soft Computing Journal, 2007, 7(2):585-592

[6] Zhang W, Guo X., Wang C., Wu C. A POD-Based Center Selection for RBF Neural Network in Time Series Prediction Problems [J]. LECTURE NOTES IN COMPUTER SCIENCE, 2007, 44(32):189.

[7] Du J. Y, Feng J. X., Su L. Research on RBF network based prediction model for alloy burdening [J]. Liangang (Steelmaking), 2007, 23(3):32-35. 